\title{
Revisiting operons: an analysis of the landscape of transcriptional units in E. coli
}

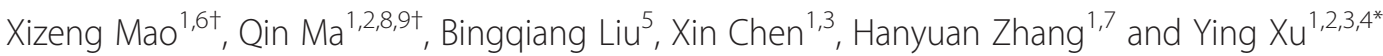

\begin{abstract}
Background: Bacterial operons are considerably more complex than what were thought. At least their components are dynamically rather than statically defined as previously assumed. Here we present a computational study of the landscape of the transcriptional units (TUs) of E. coli K12, revealed by the available genomic and transcriptomic data, providing new understanding about the complexity of TUs as a whole encoded in the genome of E. coli K12.

Results and conclusion: Our main findings include that (i) different TUs may overlap with each other by sharing common genes, giving rise to clusters of overlapped TUs (TUCs) along the genomic sequence; (ii) the intergenic regions in front of the first gene of each TU tend to have more conserved sequence motifs than those of the other genes inside the TU, suggesting that TUs each have their own promoters; (iii) the terminators associated with the $3^{\prime}$ ends of TUCs tend to be Rho-independent terminators, substantially more often than terminators of TUs that end inside a TUC; and (iv) the functional relatedness of adjacent gene pairs in individual TUs is higher than those in TUCs, suggesting that individual TUs are more basic functional units than TUCS.
\end{abstract}

Keywords: Operon, Transcriptional unit, Promoter, Terminator, Bacteria

\section{Background}

The concept of operon as a transcriptional unit (TU) was first proposed by French scientists Jacob and Monod in 1960 when they were studying the lactose metabolism in E. coli [1]. They defined an operon as a list of genes that are transcribed in a single polycistronic unit and share the same genetic regulation signals. In their seminal paper [1], Jacob and Monod proposed operons as a model to coordinately transcribe a group of genes arranged in tandem on the same genomic strand, and suggested that all genes in a bacterial cell are controlled by means of operons through a single feedback regulatory mechanism. Since then, operons have been used as the basic transcriptional and functional units in bacterial studies. Such information has been widely applied to derive higher-level functional organizations such as biochemical pathways/networks and regulation systems, which are difficult to derive in eukaryotic organisms.

\footnotetext{
* Correspondence: xyn@bmb.uga.edu

${ }^{\dagger}$ Equal contributors

${ }^{1}$ Computational Systems Biology Lab, Department of Biochemistry and Molecular Biology, and Institute of Bioinformatics, University of Georgia, Athens, USA

${ }^{2}$ BioEnergy Research Center (BESC), Athens, GA, USA

Full list of author information is available at the end of the article
}

A widely-held assumption in computational operon prediction has been that operons generally do not overlap $[2,3]$ although this has never been suggested by Jacob and Monod in their original paper [1]. This assumption allows computational predictions of operons based on sequence-level information alone, and has been popularized through the widely used operon databases such as DBTBS [4], OperonDB [5] and DOOR [6, 7], which were developed based on such an assumption. The rapidly increasing pool of large-scale transcriptomic and proteomic data collected under multiple conditions have clearly shown that this assumption is generally not true [8-10]. Specifically, different subsets of genes in an "operon" may be co-transcribed under different conditions. One such example is that the pdhR-aceEF-lpd operon in $E$. coli, consisting of four genes ( $p d h R, a c e E, a c e F, l p d)$, has at least three experimentally validated transcriptional units, i.e., the whole operon, $(a c e E$, $a c e F)$ and $(l d p)$ under different conditions [11]. The general situation is actually more complex than this as our analysis of large-scale transcriptomic data revealed that generally there may not a mother operon, of which different subsets of its genes are expressed under different conditions; instead the situation tends to be that there are multiple parallel operons, which 
may overlap but are not subsets of each other, forming a cluster of overlapping TUs along with the genomic sequence. A number of studies aiming to identify TUs revealed by specified RNA-seq data have been published such as [12-16]. We have previously developed a computer program to infer TUs based on strand-specific RNA-seq data [17]. While our initial application was done on $C$. thermocellum, the tool should be generally applicable to any bacteria.

Here we present a computational study of $E$. coli $\mathrm{K} 12$ transcriptomic data, aiming to (1) derive as many different TUs as possible based on the available transcriptomic data, and (2) study their genomic locations and regulations. Here a TU is defined as a list of genes, which is transcribed into one RNA molecule under some conditions [18]; hence an "operon" is a TU. To avoid confusions, we use TUs to represent operons as defined by Jacob and Monod, and use "operons" to refer to those computationally predicted and stored in public operon databases. A TU cluster (TUC) is defined as a maximal set of TUs such that every pair of its TUs are connected with each other, where two TUs are connected if they share common genes or they each share common genes with other TUs that are connected. Throughout the paper, a TUC is also referred to as the parent TUC of its member TUs. In addition, we have the following definitions: (A) TUs that span the entire DNA sequence covered by a TUC are referred to as full TUs; (B) starting TUs are the ones that begin with the first gene of their parent TUCs excluding (A); (C) terminal TUs are those that end with the last gene of their parent TUCs excluding (A); and (D) internal TUs are those that contain neither the first nor the last gene of their parent TUCs. TUs of (B) and (D) are called non-terminal TUs; and TUs of (C) and (D) are non-starting TUs (see Fig. 1).

Numerous TUs have been experimentally identified in E. coli K12. For example, a study by Palsson's group identified 942 TUs based on genome-scale transcriptomic data collected under four conditions [9]. The
RegulonDB contains 842 experimentally validated TUs [19]. We have integrated these datasets plus our own operon prediction in the DOOR database [20] as the currently known TUs of E. coli K12, and made a number of discoveries about TUs/TUCs and their regulatory relationships. The most interesting discoveries are that (i) terminators of the terminal TUs tend to be Rho-independent terminators, more often than those of the nonterminal TUs; (ii) the intergenic regions in front of the first genes of TUs tend to have more conserved sequence motifs than those of the other genes inside the TUs, suggesting that TUs may each have their own promoters; and (iii) the functional relatedness between adjacent genes within TUs is higher than those within the same TUCs but not the same TUs, indicating that TUs are likely more basic functional units than TUCs. Our analysis programs and the predicted TUCs are available at http://csbl.bmb.uga.edu/ xizeng/research.php?p=TU.

\section{Results}

\section{Characteristics of TUCs}

To predict TUCs encoded in the E. coli genome, we have integrated the datasets in the Palsson's paper [9] and RegulonDB database [21] along with E. coli operons in our DOOR operon database [7]. This gives rise to a total of 2,227 TUCs, including 1,342 single-gene TUCs and 885 multi-gene TUCs (Additional file 1). Figure 2 shows the size distribution of all the 885 multi-gene TUCs in terms of the number of TUs per TUC, in which 656 (74 \%) multi-gene TUCs each have at least two TUs. All the predicted TUCs can be accessed at http://csbl.bmb.u ga.edu/ xizeng/research.php?p=TU.

To study the structure of TUCs in the genome, we have compared them with directons, each of which is the maximal set of consecutive genes on the same genomic strand without genes on the opposite strand interrupting the continuity $[3,22]$. We intuitively expect that all the TUCs are each contained inside one directon, which proves to be the case based on our analyses. Overall, the

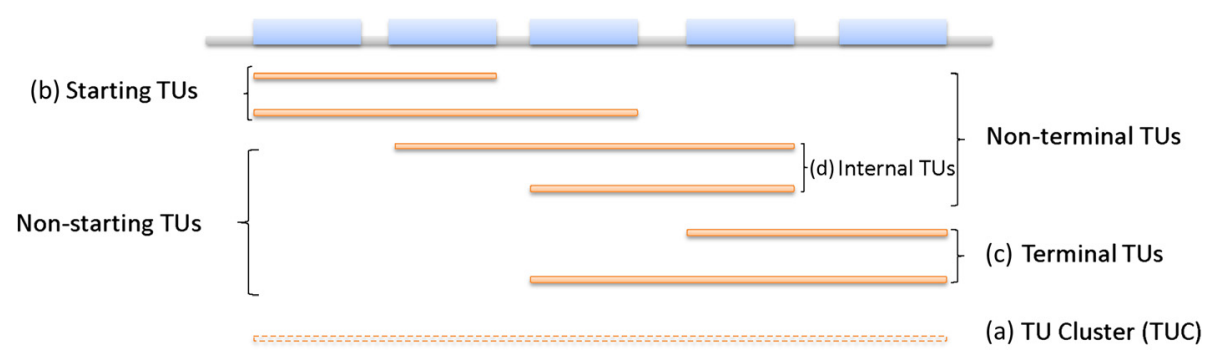

Fig. 1 A diagram of TUC and different TU types: (a) TUs that span the entire DNA sequence covered by a TUC, referred to as full TUs; (b) starting TUs are the ones that begin with the first gene of their parent TUCs excluding $(\mathbf{a})$; (c) terminal TUs are the ones that end with the last gene of their parent TUCs excluding (a); and (d) internal TUs are the ones that contain neither the first nor the last gene of their parent TUCs (see Fig. 1). TUs of (b) and (d) are called non-terminal TUs; and TUs of (c) and (d) are non-starting TUs. Blue bars represent genes, and each solid orange line represents a TU, and the dashed orange line in the bottom is a TUC 

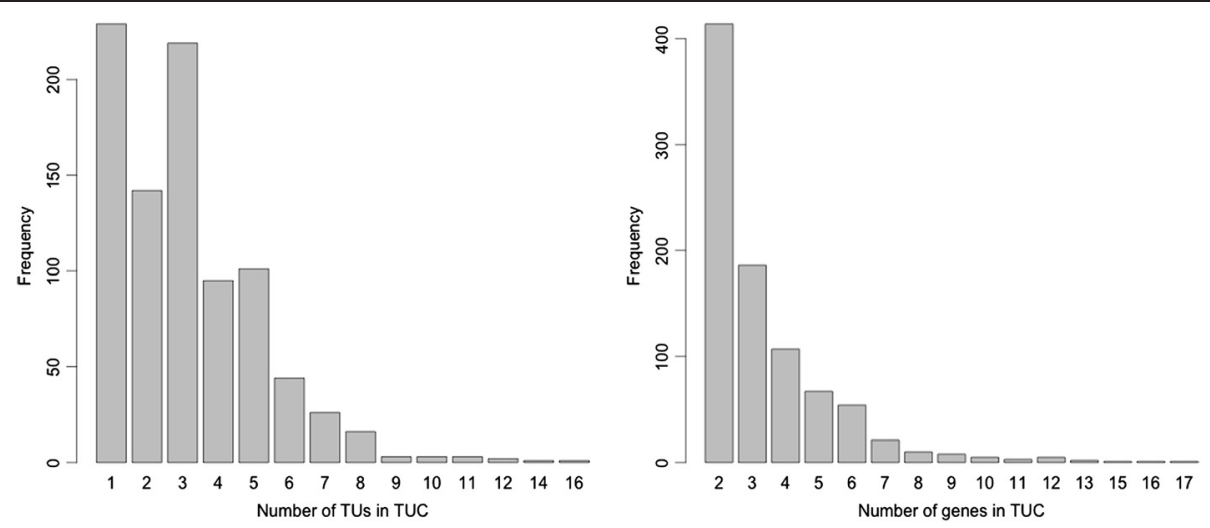

Fig. 2 Distributions of the number of genes and the number of TUs per multi-gene TUC across all 885 TUCs

E. coli genome has 1,318 directons and (at least) 2,227 TUCs. We noted that (a) 807 (36\%) TUCs match perfectly with their parent directons, i.e., sharing the boundary genes at both ends of the directons; 1,022 (46 $\%)$ TUCs share exactly one boundary gene of their parent directons; and 398 (18\%) of the TUCs are located properly inside their parent directons (see Fig. 3a); and (b) 806 of the 1,318 (61\%) directons each contain at least two TUCs (see Fig. 3b).

\section{Non-starting TUs likely have their own promoters}

To check whether individual TUs may have their own promoters, we classified all genes in TUCs into three categories: A: the set of all 5'-end genes in TUCs; B: the

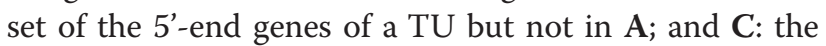
set of genes covered by at least one TU but not in (A or B). We compared the following numbers across the three categories: (i) the number of genes having known and predicted binding sites for transcription factors
(TFBS); and (ii) the number of genes having validated promoters downloaded from RegulonDB [21] (see Methods). We found that (i) B genes have more known and predicted TFBSs than $\mathbf{C}$ genes, and less than $\mathbf{A}$ genes; and (ii) B genes have substantially more validated promoters than $\mathbf{C}$ genes, and less than $\mathbf{A}$ genes (see Table 1). From these observations, we conclude that TUs are likely to use their own promoters.

To understand the differences between the $\mathbf{A}$ genes and the $\mathbf{B}$ genes, we have examined the lengths of their 5 ' upstream inter-genic regions, and compared the average lengths of the inter-genic regions in front of the $\mathbf{A}$ genes and that of the $\mathbf{B}$ genes, as well as the average numbers of confidently predicted TFBSs in such regions for the A genes versus the $\mathbf{B}$ genes. We found that the average length and the average number of TFBSs are 203 bps and 1.9 for A genes, respectively, compared to 101 and 0.5 for the $\mathbf{B}$ genes in the Palsson dataset; and 195 and 1.8 for the A genes versus 121 and 0.5 for the $\mathbf{B}$
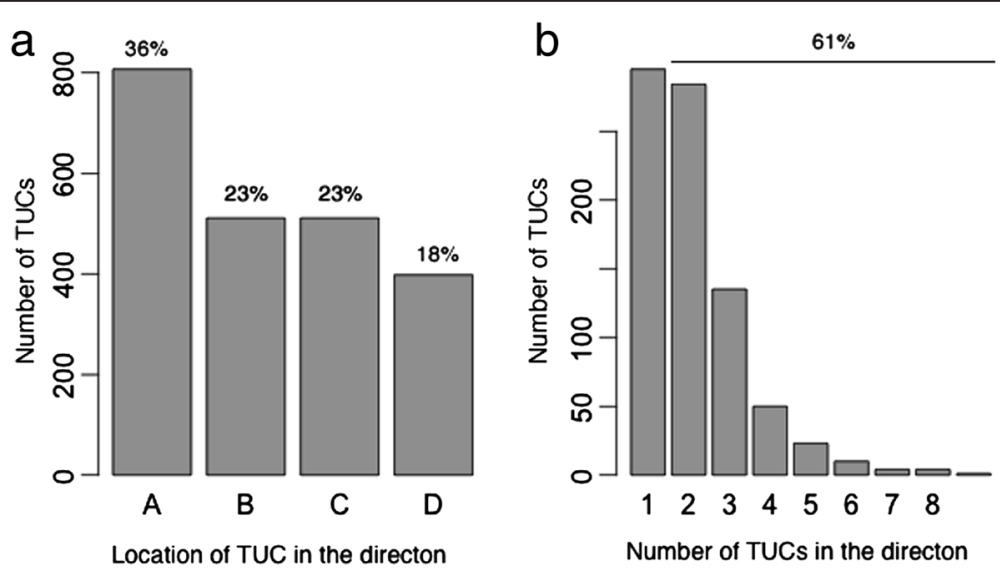

Fig. 3 Relations between TUCs and directons in the E. coli K12 genome. In (a), A represents the TUCs matching perfectly with their parent directons; $\mathbf{B}$ for the TUCs sharing exactly the $5^{\prime}$ boundary genes of their parent directons but; $\mathbf{C}$ for the TUCs sharing exactly the $3^{\prime}$ boundary genes of their parent directons; and $\mathbf{D}$ the TUCs located properly inside their parent directons. (b) The $x$-axis represents the number of TUCs per directon and the $y$-axis represents the number of directons containing $k$ TUCs for $k=1, \ldots, 8$ 
Table 1 Statistics of 5,430 conserved sequence motifs, 3,307 known plus 2,123 predicted TFBSs, and 3,754 predicted promoters for genes in $\mathbf{A}, \mathbf{B}$ and $\mathbf{C}$, respectively, with these sets defined above

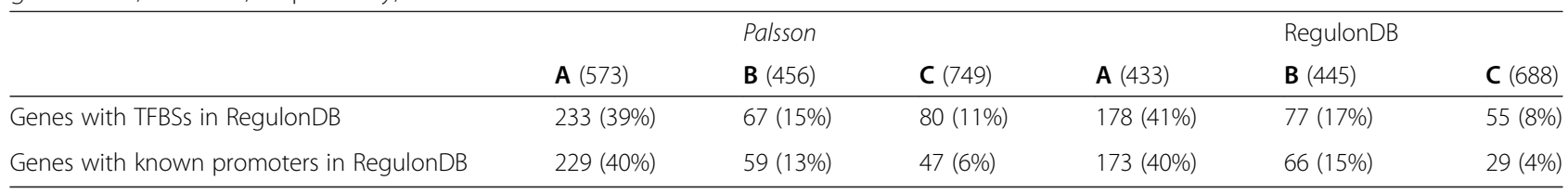

genes in RegulonDB. These data suggest that TUs starting with the A genes may serve as the default or frequently used TUs compared to the other TUs within each TUC. We then examined the over-represented Gene Ontology (GO) categories by the A, B and C genes, respectively; and found that the $\mathbf{A}$ genes do not share any of their over-represented GO categories with the ( $\mathbf{B}$ or $\mathbf{C}$ ) genes, while the $\mathbf{B}$ genes do share some of their over-represented $\mathrm{GO}$ categories with the $\mathbf{C}$ genes, suggesting that non-starting genes in a TU are functionally more relevant with each other. We also noted that these observations are highly consistent between the Palsson set and RegulonDB as summarized in Table 1, providing a cross-validation between the two datasets.

\section{Non-terminal TUs more likely use Rho-dependent terminators}

It is known that $E$. coli uses two different mechanisms for transcription termination: Rho-independent and Rhodependent termination [23]. Rho-dependent termination involves the binding of a Rho factor to an mRNA to destabilize the RNA-DNA interaction while $R$ ho-independent termination functions by creating an RNA hairpin loop to stop the RNA polymerase [24]. Rho-independent terminators can be effectively predicted based on the identification of the conserved RNA hairpin loop, while Rhodependent terminators cannot yet due to the lake of signals known to be associated with them.

To examine if different TUs may have preferences in using either type of termination, we have carried out the following analysis. Using the widely used TranstermHP program [25], we predicted 1,835 Rho-independent terminators with confidence score at least 76 for the 4,164 genes of $E$. coli, which is the cutoff for reliable predictions as suggested by the authors of the program. We define the following three sets of genes: $\mathbf{D}$ as the set of the 3'-end genes of TUCs; E: the set of the 3'-end genes of TUs but not in $\mathbf{D}$; and $\mathbf{F}$ the set of all the other genes in TUs but not in (D or $\mathbf{E}$ ). We found that (a) $\mathbf{E}$ uses far fewer Rho-independent terminators than D percentagewise; (b) F uses a fewer Rho-independent terminators than $\mathbf{E}$, as detailed in Table 2; and (c) D, E and $\mathbf{F}$ genes do not share any of their respectively over-represented GO categories (Additional file 2). These data revealed that (i) TUCs tend to end with Rho-independent terminators; (ii) TUs not using the same ends with their parent TUCs use predominantly Rho-dependent terminators; and (iii) the predicted $R h o$-independent terminators associated with the $\mathbf{F}$ genes may represent false predictions, on both the Palsson dataset and the RegulonDB.

We do note that both the D and the E sets in Palsson's dataset are substantially smaller than those in RegulonDB. We suspect that the reason is the segmentation algorithm used Palsson's study may tend to break a long TU into smaller ones, hence artificially leading to shorter TUCs and hence smaller $\mathbf{E}$ and $\mathbf{D}$. To test if this hypothesis may be true, we have examined the sizes of TUs in both the Palsson set and RegulonDB, and found that the Palsson set indeed has considerably more small TUs consisting of at most two genes, while RegulonDB has more large TUs having 3 to 6 genes, indicating that there is a systematic difference between the sizes of TUs of the two datasets (see details in Fig. 4). This provides a strong supporting evidence to our hypothesis. It is worth noting that we have ignored the larger TUs (size $\geq 7$ ) in above calculation due to their low occurrence frequency ( $\leq 5 \%)$.

Another curious issue is that 1,149 of the 2,227 3'-end genes of TUCs, denoted as $\mathbf{X}$, are not predicted to end with a Rho-independent terminator, suggesting the possibility that an overly stringent cutoff (76 as default) is used by the TranstermHP program. To test this hypothesis, we have re-run the TranstermHP program using lower cutoff values, $37,47,57$, and 67 , on genes in $\mathbf{X}$ and those non-3'-end genes of TUCs, denoted as $\mathbf{Y}$; and found that, percentage-wise, $\mathbf{X}$ has substantially more Rho-independent terminators than $\mathbf{Y}$ (see details in Fig. 5), which provides a strong supporting evidence to our hypothesis.

Table 2 Rho-independent terminators for $\mathbf{D}, \mathbf{E}$ and $\mathbf{F}$ genes, as defined above

\begin{tabular}{|c|c|c|c|c|c|c|}
\hline & & Palsson & & & RegulonD & \\
\hline Category & D (573) & E (335) & $\mathbf{F}(821)$ & E (433) & E (359) & $\mathbf{F}(765)$ \\
\hline Genes & $271(47 \%)$ & 46 (14\%) & $102(12 \%)$ & 229 (59\%) & 70 (19\%) & $65(8 \%)$ \\
\hline
\end{tabular}




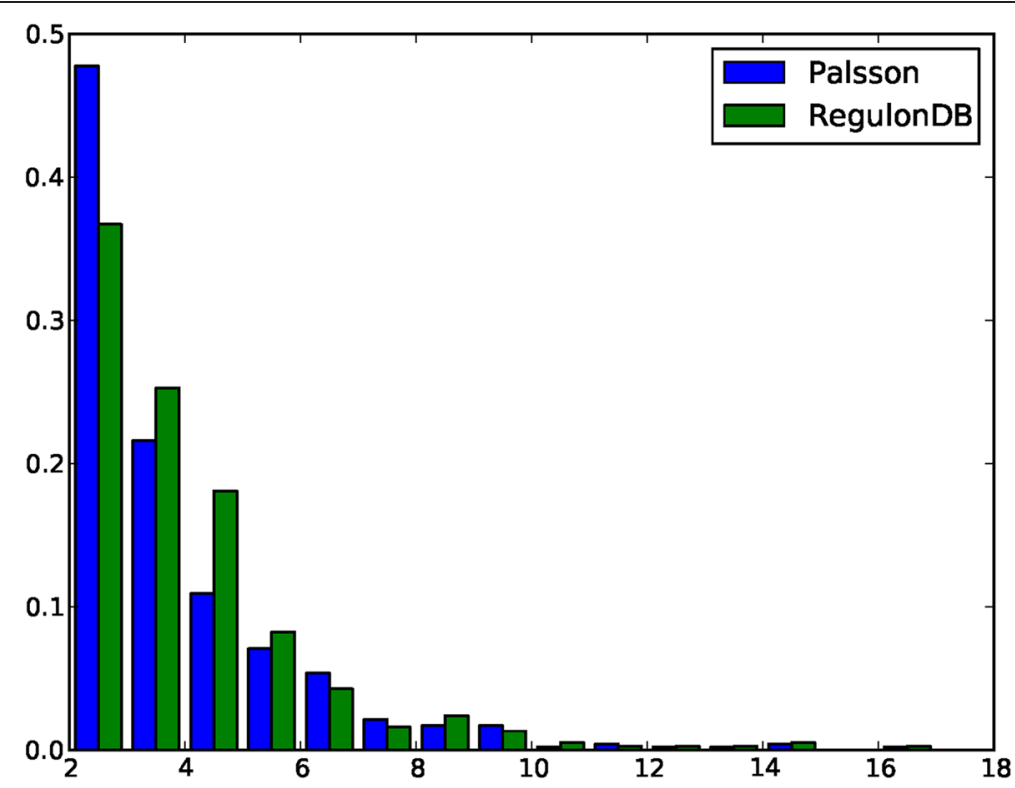

Fig. 4 Histograms of TU sizes in the Palsson and RegulonDB datasets

Individual TUs are more basic functional units than TUCs Based on our preliminary analyses, we speculate that TUs are more basic functional units than TUCs. To demonstrate this, we have examined the levels of functional relatedness and the co-occurrence conservation for three types of consecutive gene pairs: A: gene pairs each consisting of two adjacent genes in two different TUCs (omitting the cases where both TUCs being single-gene ones); B: gene pairs each consisting of a 5' gene of a TU and the gene in its immediate upstream on the same genomic strand, excluding those in $\mathbf{A}$; and $\mathbf{C}$ : all other gene pairs inside a TU. The functional relatedness of these gene pairs [26] is assessed using a combined phylogenetic profile analysis [27], gene neighborhood analysis [28] and Gene Ontology assignment [29], and the cooccurrence conservation is measured using the number of genomes in which their orthologous genes are adjacent with each other in 216 reference genomes [30] (see Methods). We find from Fig. 6 that the functional relatedness and the co-occurrence conservation level both show clear increasing trend going from the $\mathbf{A}$ to $\mathbf{B}$ to the $\mathbf{C}$ genes, which strongly suggests that TUs likely have served

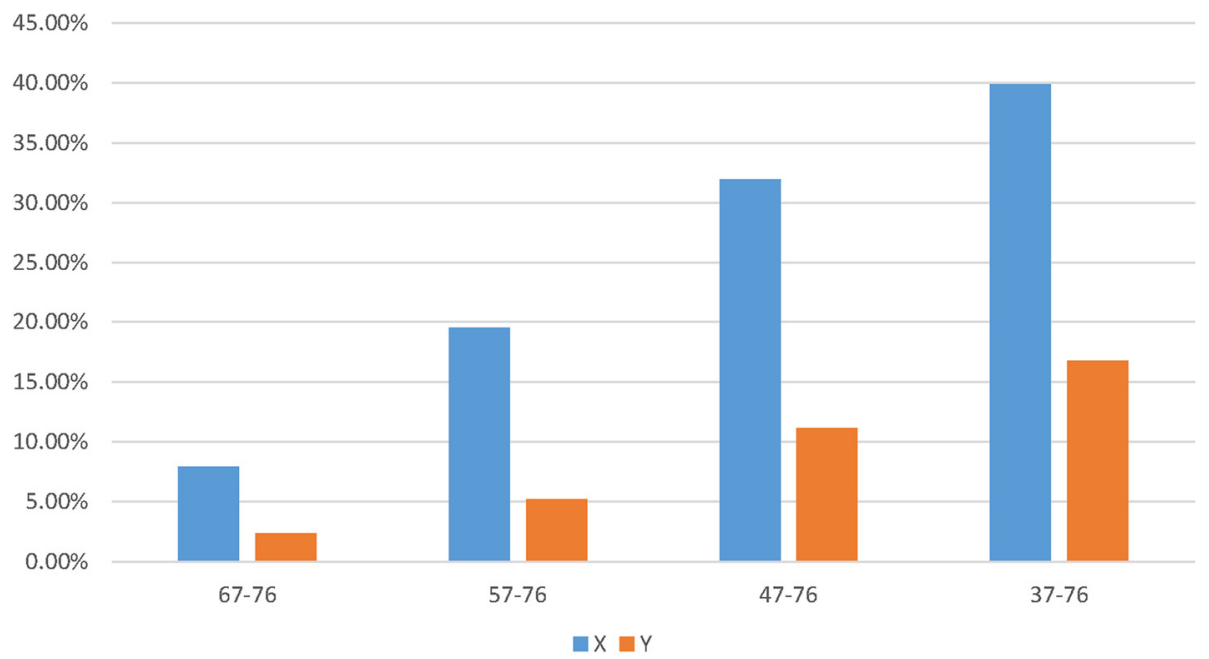

Fig. 5 The percentage of genes having Rho-independent terminators with confidence score lower than 76, predicted by the TranstermHP program. $\mathbf{X}$ represents 1,149 3'-end genes of TUCs having Rho-independent terminators with confident score no less than 76; and $\mathbf{Y}$ represents 1,919 non-3'-end genes of TUCS 


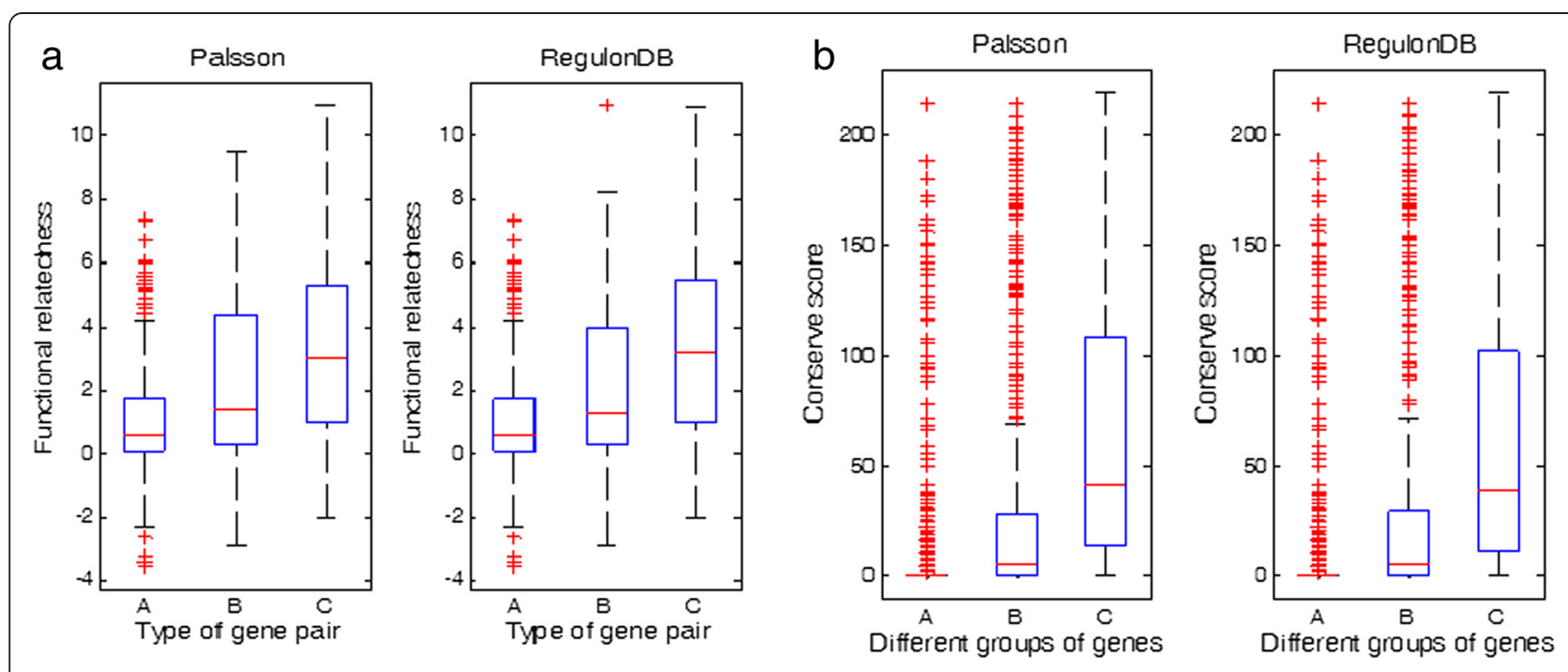

Fig. 6 Boxplots of gene functional relatedness and conservation levels for the three types of gene pairs. a Gene functional relatedness respectively for the Palsson and RegulonDB data sets; (b) gene location conservation levels respectively for the Palsson and RegulonDB data sets

as more basic functional units selected during evolution than TUCs.

\section{Discussion}

Our analyses have shown Rho-independent terminators tend to be associated with the end of a TUC, while nonterminal TUs tend to use Rho-dependent terminators. This suggests that Rho-independent terminators may be associated with the end of a cluster of functionally related genes while Rho-dependent terminators are associated with portions of TUCs, which are used under specific conditions that may trigger the release of the Rho factors.

It is noteworthy that the TUCs studied here may be smaller than the actual TUCs encoded in the E. coli K12 genome as our analysis suggests, as some of the true TUs may not be revealed under the conditions covered by Palsson's dataset and RegulonDB, which may connect two predicted TUCs into one.

To examine whether the organization of TUCs may be related to chromosomal folding, we have compared the TUCs with the predicted folding domains, called supercoils, of the E. coli $\mathrm{K} 12$ genome, which typically each range from $15 \mathrm{Kbps}$ to $100 \mathrm{Kbps}$ in length, and the two ends of each supercoil join together through binding with nucleoid associated proteins (NAPs) [31-33] such as H-NS and Fis $[34,35]$ in a folded chromosome. It has been observed that supercoils may be condition-dependent, i.e., a different set of supercoils may be formed under different conditions [36]. Other than such binding information, no genomescale supercoil boundary data have been published. We have previously predicted 409 putative supercoils, along with 409 boundary regions in the circular genome of $E$. coli $K 12$ based on 527 experimentally validated binding sites of the NAP proteins [32]. We found that 148 out of the 1,078
$(606+472)$ TUCs ending with Rho-independent terminators have their 3' ends coincide with (predicted) supercoil boundary regions, and 91 out of the remaining 1,149 TUCs ending with Rho-dependent terminators have their 3'-ends coincide with supercoil boundary regions. We have also examined the average gene-expression level of TUCs in the different locations of supercoils under the 466 experimental conditions in the M3D database [37], and found that the TUCs at the supercoil boundaries have higher average gene expression level (with $P$-value $1.1 \mathrm{e}-4$ by the Wilcox test) than those in the middle (Additional file 3). The statistical significance in achieving this level of coincidence for the two cases are 1e-6 and 0.01, respectively, suggesting that supercoil boundaries may play some role in determining the organization of TUCs.

\section{Conclusion}

We have presented a computational study of the landscape of the TUs encoded in the genome of E. coli K12, revealed by the available transcriptomic data, and shown new understanding about the organization of TUs as a whole encoded in the genome of $E$. coli K12. Our main findings are: (i) different TUs may overlap with each other by sharing common genes, giving rise to clusters of overlapped TUs, i.e.,TUCs; (ii) the intergenic regions in front of the first genes of TUs tend to have more conserved sequence motifs than those of the other genes inside the TUs, suggesting that TUs each likely have their own promoters; (iii) the terminators associated with the 3'-ends of TUCs tend to be Rho-independent terminators, considerably more often than terminators of non-terminal TUs; and (iv) the functional relatedness of adjacent gene pairs within TUs is higher than those in the same TUCs but not in the same TUs, indicating that TUs are likely more basic 
functional units than TUCs during evolution. To the best of our knowledge, this is the first systemic and large-scale study of the general properties of TUs and TUCs. We anticipate that the knowledge gained here will prove to be useful to scientists who study bacterial genomes, transcription and evolution.

\section{Methods}

Data

E. coli operons used in this study were downloaded from the DOOR operon database at http://csbl.bmb.uga.edu/ DOOR/. A total of 2,325 operons are predicted for E. coli $\mathrm{K} 12$, which includes 884 multi-gene operons covering 2,704 genes and 1,441 single-gene operons. Based on comparisons with experimentally validated operons, the predicted multi-gene operons have an accuracy level at $93.7 \%$ [20].

We have downloaded a dataset of 942 TUs from Palsson's paper [9] (http://gcrg.ucsd.edu/InSilicoOrganisms/Ecoli) and 842 TUs from the RegulonDB database [19]. The two datasets share 398 common TUs, which is not surprising since TUs are condition-dependent and these two datasets are collected under different conditions. The relatively small overlap between the two sets also suggest that a large number of TUs are not covered by either of these two sets.

2,237 known and 1,770 predicted transcription factor binding sites, 3,754 promoters of E. coli are collected from the RegulonDB database [19]. The TranstermHP program [25] was used to predict Rho-independent terminators in $E$. coli, which has a prediction sensitivity at $89 \%$ and specificity at $98 \%$ for B. subtilis according to the authors of the program. For each TUC without a Rho-independent terminator, we consider that it has a Rho-dependent terminator.

We downloaded the Gene Ontology categories for $E$. coli from the org.EcK12.eg.db $\mathrm{R}$ package and used the GOstats R package to identify the over-represented categories given a set of genes based on the hypergeometric distribution.

We have predicted 409 supercoil domains and the same number of their boundary regions in the (circular) E. coli K12 chromosome [32] using 347 metabolic pathways from EcoCyc [38] and genome-scale geneexpression data collected under 466 conditions in the M3D database [37], based on the following hypothesis: the chromosome of E. coli is partitioned into a set of contiguous and independent folding domains under specific growth conditions so that the total number of unfolding of such domains is minimized to make their genes transcriptionally accessible [39]. We then formulated the domain boundary prediction problem as a genome-partition optimization problem and solved it using a dynamic programming approach [32].

\section{Identification of TU clusters}

We have used the two sets of TUs described in Introduction and the 2,325 predicted operons in the DOOR database to predict the TUCs. Overall 4,139 distinct TUs are considered here. We represent each TU as a vertex in a graph, a pair of TUs is connected by an un-weighted edge if they overlap, and each TU Cluster as a maximal connected component. We thus identify each maximal connected component in a graph as a TUC using an in-house Perl script that is accessible on the web page http:// csbl.bmb.uga.edu/ xizeng/research.php?p=TU.

\section{Analysis of functional relatedness of gene pairs}

The functional relatedness of gene pairs are accessed from [26], which incorporates phylogenetic profile analysis [27], gene neighborhood analysis [28] and Gene Ontology assignment [29]. Meanwhile, the co-occurrence conservation level of a gene pair is measured by the number of species in which their orthologous genes are adjacent in a list of 216 reference genomes, which are selected within the same phylum but in different genus of $E$. coli, called reference species (Released on 2011-11-01, NCBI). In each genus, we selected the largest genome to avoid potential selection bias in comparative genomics studies [40]. The GOST program [41] is used to identify the orthologous genes of each E. coli gene across the 216 reference genomes.

\section{Additional files}

Additional file 1: The identified TUCs and their properties and characteristics. (XLSX $258 \mathrm{~kb}$ )

Additional file 2: The GO functional enrichment analysis. (XLSX $99 \mathrm{~kb}$ )

Additional file 3: Figure S1. The comparison of expression values between boundary genes and internal genes. (DOCX $101 \mathrm{~kb}$ )

\section{Abbreviations}

DBTBS: database of transcriptional regulation in B. subtilis; DOOR: database of prokaryotic operons; E. coli: Escherichia coli; EcoCyc: a comprehensive database resource for Escherichia coli; GOST: global optimization strategy; NAP: nucleoid associated proteins; NCBI: National Center for Biotechnology Information; RegulonDB: regulon database; TU: transcriptional unit;

TUC: transcriptional unit cluster.

\section{Competing interests}

The authors declare that they have no competing interests.

\section{Authors' contributions}

YX conceived the study, participated in its design and coordination, and drafted the manuscript. QM and XM participated in the coordination of the paper, carried out or participated all the analyses of TU and TU clusters, and drafted the manuscript. $B L$ analyzed the relation of Rho-dependent terminator and TU. XC participated in the promoter study of TU. HZ carried out the implementation of TU cluster identification algorithm. All authors read and approved the final manuscript.

\section{Acknowledgement}

We thank all the members of the CSBL Lab at UGA, especially Dr. Yanbin Yin for discussion of TU transcription regulation and Dr. Victor Olman for the discussion of statistics, Mr. Kan Bao for discussion of statistical methods, Mr. 
Henry Schwartz for help of promoter study of TU, and Ms. Lauren Regan for help of data preparation. This work is funded by National Science Foundation [NSF MCB-0958172]; and DOE BioEnergy Science Center, supported by the Office of Biological and Environmental Research in the Department of Energy Office of Science [DE-PS02-06ER64304]. This work was also supported in part by the Agriculture Experiment Station (SDOOH558-15) and the Biochemical Spatiotemporal Network Resource Center (3SP680) of South Dakota State University.

\section{Author details}

'Computational Systems Biology Lab, Department of Biochemistry and Molecular Biology, and Institute of Bioinformatics, University of Georgia, Athens, USA. ${ }^{2}$ BioEnergy Research Center (BESC), Athens, GA, USA. ${ }^{3}$ College of Computer Sciences and Technology, Changchun, Jilin, China. ${ }^{4}$ School of Public Health, Jilin University, Changchun, Jilin, China. ${ }^{5}$ School of Mathematics, Shandong University, Jinan, Shandong, China. ${ }^{6}$ Present address: MD Anderson Cancer Center, Houston, TX 77054, USA. ${ }^{7}$ Present address: Systems Biology and Biomedical Informatics (SBBI) Laboratory University of Nebraska-Lincoln 122B/122C Avery Hall, 1144 T St, Lincoln, NE 68588-0115, USA. ${ }^{8}$ Present address: Department of Plant Science, South Dakota State University, Brookings, SD 57006, USA. ${ }^{9}$ Present address: BioSNTR, Brookings, SD, USA.

Received: 22 July 2015 Accepted: 29 October 2015 Published online: 04 November 2015

\section{References}

1. Jacob F, Perrin D, Sanchez C, Monod J. Operon: a group of genes with the expression coordinated by an operator. C R Hebd Seances Acad Sci. 1960;250:1727-9.

2. Craven M, Page D, Shavlik J, Bockhorst J, Glasner J. A probabilistic learning approach to whole-genome operon prediction. Proceedings/ International Conference on Intelligent Systems for Molecular Biology. 2000;8:116-27.

3. Salgado H, Moreno-Hagelsieb G, Smith TF, Collado-Vides J. Operons in Escherichia coli: genomic analyses and predictions. Proceedings of the National Academy of Sciences of the United States of America. 2000;97(12):6652-7.

4. Sierro N, Makita Y, de Hoon M, Nakai K. DBTBS: a database of transcriptional regulation in Bacillus subtilis containing upstream intergenic conservation information. Nucleic acids research. 2008;36(Database issue):D93-96.

5. Pertea M, Ayanbule K, Smedinghoff M, Salzberg SL. OperonDB: a comprehensive database of predicted operons in microbial genomes. Nucleic acids research. 2009;37(Database issue):D479-482.

6. Mao F, Dam P, Chou J, Olman V, Xu Y. DOOR: a database for prokaryotic operons. Nucleic acids research. 2009;37(Database issue):D459-463.

7. Mao X, Ma Q, Zhou C, Chen X, Zhang H, Yang J, et al. DOOR 2.0: presenting operons and their functions through dynamic and integrated views. Nucleic acids research. 2013;42(Database issue):D654-9.

8. Koide T, Reiss DJ, Bare JC, Pang WL, Facciotti MT, Schmid AK, et al. Prevalence of transcription promoters within archaeal operons and coding sequences. Molecular systems biology. 2009;5:285.

9. Cho BK, Zengler K, Qiu Y, Park YS, Knight EM, Barrett CL, et al. The transcription unit architecture of the Escherichia coli genome. Nature biotechnology. 2009;27(11):1043-9.

10. Quax TE, Wolf Yl, Koehorst JJ, Wurtzel O, van der Oost R, Ran W, et al. Differential translation tunes uneven production of operon-encoded proteins. Cell reports. 2013:4(5):938-44.

11. Quail MA, Haydon DJ, Guest JR. The pdhR-aceEF-Ipd operon of Escherichia coli expresses the pyruvate dehydrogenase complex. Mol Microbiol. 1994;12(1):95-104.

12. Conway T, Creecy JP, Maddox SM, Grissom JE, Conkle TL, Shadid TM, et al. Unprecedented high-resolution view of bacterial operon architecture revealed by RNA sequencing. mBio. 2014;5(4):e01442-01414.

13. Chen $\mathrm{H}$, Shiroguchi $\mathrm{K}$, Ge H, Xie XS. Genome-wide study of mRNA degradation and transcript elongation in Escherichia coli. Molecular systems biology. 2015;11(1):781

14. Li S, Dong X, Su Z. Directional RNA-seq reveals highly complex conditiondependent transcriptomes in E. coli K12 through accurate full-length transcripts assembling. BMC genomics. 2013;14:520.
15. McClure R, Balasubramanian D, Sun Y, Bobrovskyy M, Sumby P, Genco CA, et al. Computational analysis of bacterial RNA-Seq data. Nucleic acids research. 2013;41(14):e140.

16. Raghavan R, Groisman EA, Ochman H. Genome-wide detection of novel regulatory RNAs in E. coli. Genome research. 2011;21(9):1487-97.

17. Chou WC, Ma Q, Yang S, Cao S, Klingeman DM, Brown SD, et al. Analysis of strand-specific RNA-seq data using machine learning reveals the structures of transcription units in Clostridium thermocellum. Nucleic acids research. 2015;43(10):e67.

18. Case ST, Daneholt B. The size of the transcription unit in Balbiani ring 2 of Chironomus tentans as derived from analysis of the primary transcript and 75 S RNA. Journal of molecular biology. 1978;124(1):223-41.

19. Gama-Castro S, Jimenez-Jacinto V, Peralta-Gil M, Santos-Zavaleta A, Penaloza-Spinola MI, Contreras-Moreira B, et al. RegulonDB (version 6.0): gene regulation model of Escherichia coli K-12 beyond transcription, active (experimental) annotated promoters and Textpresso navigation. Nucleic acids research. 2008;36(Database issue):D120-124.

20. Dam P, Olman V, Harris K, Su Z, Xu Y. Operon prediction using both genome-specific and general genomic information. Nucleic acids research. 2007;35(1):288-98.

21. Salgado H, Peralta-Gil M, Gama-Castro S, Santos-Zavaleta A, Muniz-Rascado L, Garcia-Sotelo JS, et al. RegulonDB v8.0: omics data sets, evolutionary conservation, regulatory phrases, cross-validated gold standards and more. Nucleic acids research. 2013;41(Database issue):D203-213.

22. Li G, Che D, Xu Y. A universal operon predictor for prokaryotic genomes. J Bioinform Comput Biol. 2009;7(1):19-38.

23. Watson JD. Molecular biology of the gene. 6th ed. San Francisco: Pearson/ Benjamin Cummings; 2008

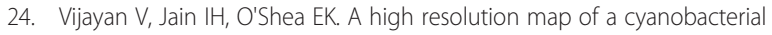
transcriptome. Genome biology. 2011;12(5):R47.

25. Ermolaeva MD, Khalak HG, White O, Smith HO, Salzberg SL. Prediction of transcription terminators in bacterial genomes. Journal of molecular biology. 2000;301(1):27-33.

26. Wu H, Su Z, Mao F, Olman V, Xu Y. Prediction of functional modules based on comparative genome analysis and Gene Ontology application. Nucleic acids research. 2005;33(9):2822-37.

27. Pellegrini M, Marcotte EM, Thompson MJ, Eisenberg D, Yeates TO. Assigning protein functions by comparative genome analysis: protein phylogenetic profiles. Proceedings of the National Academy of Sciences of the United States of America. 1999;96(8):4285-8.

28. Rogozin IB, Makarova KS, Murvai J, Czabarka E, Wolf YI, Tatusov RL, et al. Connected gene neighborhoods in prokaryotic genomes. Nucleic acids research. 2002;30(10):2212-23.

29. Ashburner M, Ball CA, Blake JA, Botstein D, Butler H, Cherry JM, et al. Gene ontology: tool for the unification of biology. The Gene Ontology Consortium. Nature genetics. 2000;25(1):25-9.

30. Alba MM, Das R, Orengo CA, Kellam P. Genomewide function conservation and phylogeny in the Herpesviridae. Genome research. 2001;11(1):43-54.

31. Benza VG, Bassetti B, Dorfman KD, Scolari VF, Bromek K, Cicuta P, et al. Physical descriptions of the bacterial nucleoid at large scales, and their biological implications. Reports on progress in physics Physical Society. 2012;75(7):076602.

32. Ma Q, Yin Y, Schell MA, Zhang H, Li G, Xu Y. Computational analyses of transcriptomic data reveal the dynamic organization of the Escherichia coli chromosome under different conditions. Nucleic acids research. 2013;41(11):5594-603.

33. Ma Q, Xu Y. Global genomic arrangement of bacterial genes is closely tied with the total transcriptional efficiency. Genomics, proteomics \& bioinformatics. 2013;11(1):66-71.

34. Luijsterburg MS, Noom MC, Wuite GJ, Dame RT. The architectural role of nucleoid-associated proteins in the organization of bacterial chromatin: a molecular perspective. Journal of structural biology. 2006;156(2):262-72.

35. Zhao Q-Y, Wang Y, Kong Y-M, Luo D, Li X, Hao P. Optimizing de novo transcriptome assembly from short-read RNA-Seq data: a comparative study. BMC bioinformatics. 2011;12 Suppl 14:S2.

36. Dillon SC, Dorman CJ. Bacterial nucleoid-associated proteins, nucleoid structure and gene expression. Nat Rev Microbiol. 2010;8(3):185-95.

37. Faith JJ, Driscoll ME, Fusaro VA, Cosgrove EJ, Hayete B, Juhn FS, et al. Many Microbe Microarrays Database: uniformly normalized Affymetrix compendia with structured experimental metadata. Nucleic acids research. 2008;36(Database issue):D866-870. 
38. Karp PD, Riley M, Saier M, Paulsen IT, Collado-Vides J, Paley SM, et al. The EcoCyc Database. Nucleic acids research. 2002;30(1):56-8.

39. Yin Y, Zhang H, Olman V, Xu Y. Genomic arrangement of bacterial operons is constrained by biological pathways encoded in the genome. Proceedings of the National Academy of Sciences of the United States of America. 2010;107(14):6310-5.

40. Che D, Li G, Mao F, Wu H, Xu Y. Detecting uber-operons in prokaryotic genomes. Nucleic acids research. 2006;34(8):2418-27.

41. Li G, Ma Q, Mao X, Yin Y, Zhu X, Xu Y. Integration of sequence-similarity and functional association information can overcome intrinsic problems in orthology mapping across bacterial genomes. Nucleic acids research. 2011;39(22):e150.

\section{Submit your next manuscript to BioMed Central} and take full advantage of:

- Convenient online submission

- Thorough peer review

- No space constraints or color figure charges

- Immediate publication on acceptance

- Inclusion in PubMed, CAS, Scopus and Google Scholar

- Research which is freely available for redistribution 